\title{
Long non-coding RNA LINC00857 promotes gastric cancer cell proliferation and predicts poor patient survival
}

\author{
KE PANG, MAO-JUAN RAN, FAN-WEN ZOU, TIAN-WEN YANG and FEI HE \\ Department of Geriatrics, Yongchuan Hospital, Chongqing Medical University, Chongqing 402160, P.R. China \\ Received February 14, 2017; Accepted January 12, 2018
}

DOI: $10.3892 / \mathrm{ol} .2018 .8883$

\begin{abstract}
Gastric cancer (GC) is a common malignancy worldwide and its pathogenesis remains unclear. Long non-coding RNAs (lncRNAs) serve an important function in cancer development, therefore identification of functional lncRNAs in GC is required. The results of the present study demonstrate that an IncRNA, LINC00857, was increased in GC tissues compared with adjacent non-tumor tissues. Overexpression of LINC00857 was positively associated with poor survival rate, as well as with the tumor size of patients with GC. LINC00857 knockdown induced by specific small interfering RNAs significantly inhibited GC cell proliferation in vitro. Genome-wide analysis revealed that LINC00857 knockdown deregulated the cell cycle. Western blot analysis confirmed that LINC00857 knockdown decreased protein expression of cyclin D1 and cyclin E1 in GC cells. Taken together, the results indicated that LINC00857 knockdown suppressed GC cell proliferation through deregulating the cell cycle, resulting in the downregulation of cyclin D1 and cyclin E1. Therefore, LINC00857 expression may be an independent biomarker for the diagnosis and prognosis of GC.
\end{abstract}

\section{Introduction}

Gastric cancer (GC) leads to a major health burden worldwide (1). Approximately 679,100 novel cases and 498,000 mortalities were estimated in China in 2015 (2). Although the incidence and mortality rates have declined in the West, the overall 5-year survival rate remains at $20 \%$ (3). The need for an effective biomarker for the diagnosis of early asymptomatic GC remains challenging (4). In current clinical practices, several serum tumor markers, including CA (carbohydrate antigen) 19-9, carcinoembryonic antigen and CA125, have been widely used, but their specificity is ambiguous (5).

Correspondence to: Dr Fei He, Department of Geriatrics, Yongchuan Hospital, Chongqing Medical University, 439 Xuanhua Road, Yongchuan, Chongqing 402160, P.R. China

E-mail: hefei1874@21cn.com

Key words: long non-coding RNAs, gastric cancer, proliferation, cell cycle
Furthermore, the current treatment options for advanced GC lack specificity, resulting in a large number of side effects and drug resistance. Thus, there is an urgent requirement to investigate novel biomarkers and therapeutic targets for GC.

Long non-coding RNAs (lncRNAs) are a class of non-coding RNAs of length $>200$ nucleotides that do not encode proteins (6). Previous studies have indicated the important function of lncRNAs in cancer development $(7,8)$. Sun et al (9) reported that the lncRNA GClncl acted as a scaffold to recruit the WDR5 and KAT2A complex and regulated the transcription of target genes. Additionally, the lncRNA HOXA11-AS acts as a scaffold to link chromatin modification factors (PRC2, LSD1 and DNMT1), and promoted cell proliferation and invasion in GC (10), suggesting that these lncRNAs may serve as therapeutic targets in GC. Genome-wide analysis has revealed that lncRNAs exhibit tissue-specific and cancer-specific expression patterns $(11,12)$. A recent study has identified several novel circulating lncRNAs for the diagnosis of GC, using genome-wide lncRNA microarrays (13). One of those lncRNAs was LINC00857, whose expression was increased in the tumor tissue and in the serum of patients with GC. In addition, LINC00857 was upregulated in patients with lung cancer and was able to promote cell proliferation, colony formation and invasion (14). However, the underlying molecular mechanism of LINC00857 in GC development remains unclear.

In the present study, it was demonstrated that LINC00857 expression was increased in GC tissues and associated with poor patient survival rates. Overexpression of LINC00857 in GC cells promoted cell proliferation and migration, suggesting that LINC00857 may be used as a novel biomarker and therapeutic target for GC.

\section{Materials and methods}

Patients and clinical samples. A total of 60 patients with GC were enrolled in the present study, which complied with the Ethics Review Board at the Yongchuan Hospital of Chongqing Medical University (Chongqing, China). Written informed consent was obtained from all patients. In total, 60 pairs of fresh GC tissues and adjacent non-tumor tissues were collected from patients who had not undergone any treatment at the Yongchuan Hospital of Chongqing Medical University between April 2012 and December 2015. The tissue samples were collected in the operating room and processed 
within $15 \mathrm{~min}$, and the non-tumorous samples were taken at a distance of $>5 \mathrm{~cm}$ from the tumor. Clinical features of patients with GC included in the present study are presented in Table I.

Cell culture. Four GC cell lines (AGS, BGC-823, MKN-45 and SGC-7901) and a gastric epithelial cell line (GES-1) were purchased from BeNa Culture Collection (Beijing China). AGS cells were cultured in Ham's F12 medium (HyClone, GE Healthcare Life Sciences, Logan, UT, USA) supplemented with 10\% fetal bovine serum (FBS; Gibco, Thermo Fisher Scientific, Inc., Waltham, MA, USA), and the remaining cell lines were cultured in Dulbecco's modified Eagle's medium/high glucose medium (HyClone; GE Healthcare Life Sciences) supplemented with $10 \% \mathrm{FBS}$ at $37^{\circ} \mathrm{C}$ in $5 \%$ $\mathrm{CO}_{2}$.

Reverse transcription-quantitative polymerase chain reaction (RT-qPCR). Total RNA was isolated from tissue and cell samples using TRIzol reagent (Thermo Fisher Scientific, Inc.), according to the manufacturer's protocol. Total RNA was resuspended in $50 \mu 1$ pre-heated $\left(65^{\circ} \mathrm{C}\right)$ nuclease-free water, subpackaged into two nuclease-free tubes and stored at $-80^{\circ} \mathrm{C}$. A PrimeScript RT reagent kit (Takara Biotechnology Co., Ltd., Dalian, China) and a Permix Ex Taq kit (Takara Biotechnology Co., Ltd.) were used to perform RT-qPCR assays according to the manufacturer's protocol. Briefly, cDNA was synthesized in a $20 \mu \mathrm{l}$ reaction volume containing $4 \mu \mathrm{l} 5 \mathrm{X}$ RT buffer, $4 \mu \mathrm{l}$ nuclease-free water, $1 \mu 1$ Prime reverse transcriptase, $1 \mu \mathrm{l}$ RT primer mixture and $10 \mu \mathrm{l}$ total RNA following genomic DNA removal at $37^{\circ} \mathrm{C}$ for $15 \mathrm{~min}$. qPCR experiments were performed in a $20 \mu 1$ reaction volume on a Bio-Rad IQ5 thermocycler (Bio-Rad Laboratories, Inc., Hercules, CA, USA) with the parameters: $95^{\circ} \mathrm{C}$ for $2 \mathrm{~min}, 40$ cycles of $95^{\circ} \mathrm{C}$ for $15 \mathrm{sec}$ and $60^{\circ} \mathrm{C}$ for $30 \mathrm{sec}$. The primer sequences for LINC00857 were as follows: 5'-CCCCTGCTTCATTGTTTCCC-3' (forward) and 5'-AGCTTGTCCTTCTTGGGTACT-3' (reverse). The primer sequences for GAPDH were as follows: 5'-GGTGGTCTC CTCTGACTTCAACA-3' (forward) and 5'-TCTCTTCCTCTT GTGCTCTTGCT-3' (reverse). LINC00857 expression was normalized using the $2^{-\Delta \Delta C q}$ method (15) relative to the mRNA expression of GAPDH.

Western blotting. SGC-7901 cells $\left(5 \times 10^{5}\right)$ were seeded in 6-well plates and transfected with small interfering RNAs (siRNAs). After $24 \mathrm{~h}$, the cells were harvested and lysed in radioimmunoprecipitation assay buffer (Roche Diagnostics, Basel, Switzerland). Total proteins were measured using a BCA kit (Thermo Fisher Scientific, Inc.), and $20 \mu \mathrm{g}$ was loaded for $12 \%$ SDS-PAGE and separated, followed by transferred onto polyvinylidene difluoride (PVDF) membranes and blocked in PBS containing 3\% bovine serum albumin at room temperature for $1 \mathrm{~h}$. Anti-F cyclin D1 rabbit (dilution, 1:500; cat. no. 2978), anti-cyclin E1 mouse (dilution, 1:500; cat. no. 4129) and anti-GAPDH rabbit (dilution, 1:1,000; cat. no. 5174) antibodies (Cell Signaling Technology, Inc., Danvers, MA, USA) were incubated at $4^{\circ} \mathrm{C}$ overnight. The goat-anti-rabbit (cat. no. ZB-2301) or goat-anti-mouse (cat. no. ZB-2305) HRP-conjugated secondary antibodies (1:10,000; ZSGB-BIO, Beijing, China) were incubated at room temperature for $1 \mathrm{~h}$. Finally, the PVDF membranes were
Table I. Clinical characterization of patients with GC.

\begin{tabular}{lc}
\hline Variable & Gastric cancer, n (\%) \\
\hline Sex & \\
Male & $36(60)$ \\
Female & $24(40)$ \\
Age, years & \\
$\geq 57$ & $31(52)$ \\
$\leq 57$ & $29(48)$ \\
Histology & \\
Adenocarcinoma & $52(87)$ \\
Mucinous adenocarcinoma & $8(13)$ \\
TNM stage & \\
I/II & $18(30)$ \\
III/IV & $42(70)$ \\
Lymph node metastasis & \\
Yes & $47(78)$ \\
No & $13(22)$
\end{tabular}

TNM, TNM classification of malignant tumors.

visualized using SuperSignal West Dura Extended Duration Substrate kit (Thermo Fisher Scientific, Inc.).

Oligonucleotide transfection. SGC-7901 cells $\left(5 \times 10^{5}\right)$ and MKN 45 cells $\left(5 \times 10^{5}\right)$ were seeded in 6-well plates, incubated for $24 \mathrm{~h}$, and then $200 \mathrm{ng} / \mathrm{ml}$ specific siRNAs for LINC00857 or negative control (NC) were transfected into the cells using Lipofectamine ${ }^{\circledR} 2000$ (Invitrogen; Thermo Fisher Scientific, Inc.) according to the manufacturer's protocol, and the cells were harvested after $24 \mathrm{~h}$. The sequences of siRNAs were as follows: siRNA1, 5'-GGUAAGGGAAGGUGGAGAAUU-3'; siRNA2, 5'-GGCUAUGUGCUGUGAACAAUU-3'.

Cell proliferation. SGC-7901 and MKN-45 cells transfected with LINC00857 siRNAs or NC were harvested and diluted at a density of $10^{4}$ cells $/ \mathrm{ml}$, prior to seeding in 96-well plates. Cell proliferation was analyzed using a Cell Counting Kit- 8 kit (CCK-8; Beyotime Institute of Biotechnology, Haimen, China) according to the manufacturer's protocol. Absorbance at $450 \mathrm{~nm}$ was measured when the reagent reacted for 24,48 , 72 or $96 \mathrm{~h}$ at room temperature.

Statistical analysis. Data were analyzed using SPSS software (version 19.0; IBM Corp., Armonk, NY, USA) and GraphPad Prism (version 6.0; GraphPad Software, Inc., La Jolla, CA, USA). All data are presented as the mean \pm standard deviation. The differences between two groups were analyzed using the unpaired Student's t-test. One-way analysis of variance with post hoc comparison using Sidak's multiple comparisons test was used for multiple groups. Patient survival was calculated using the Kaplan-Meier estimator method. $\mathrm{P}<0.05$ was considered to indicate a statistically significant difference. 
A

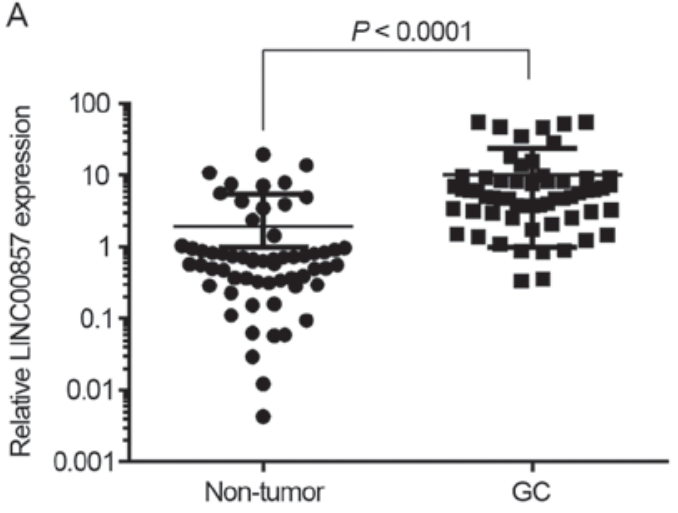

C

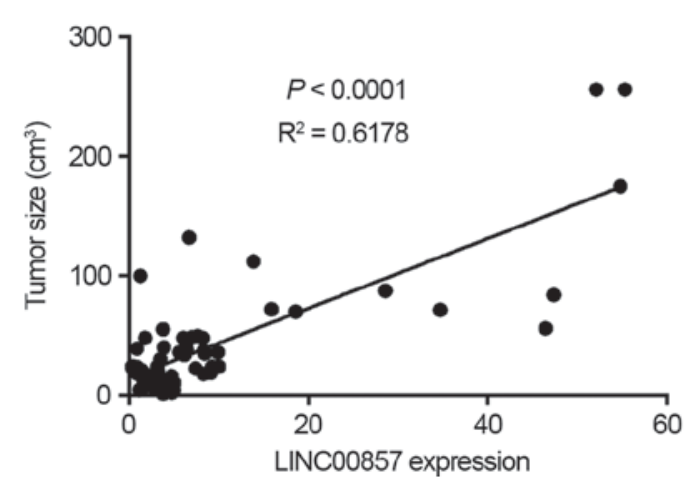

B
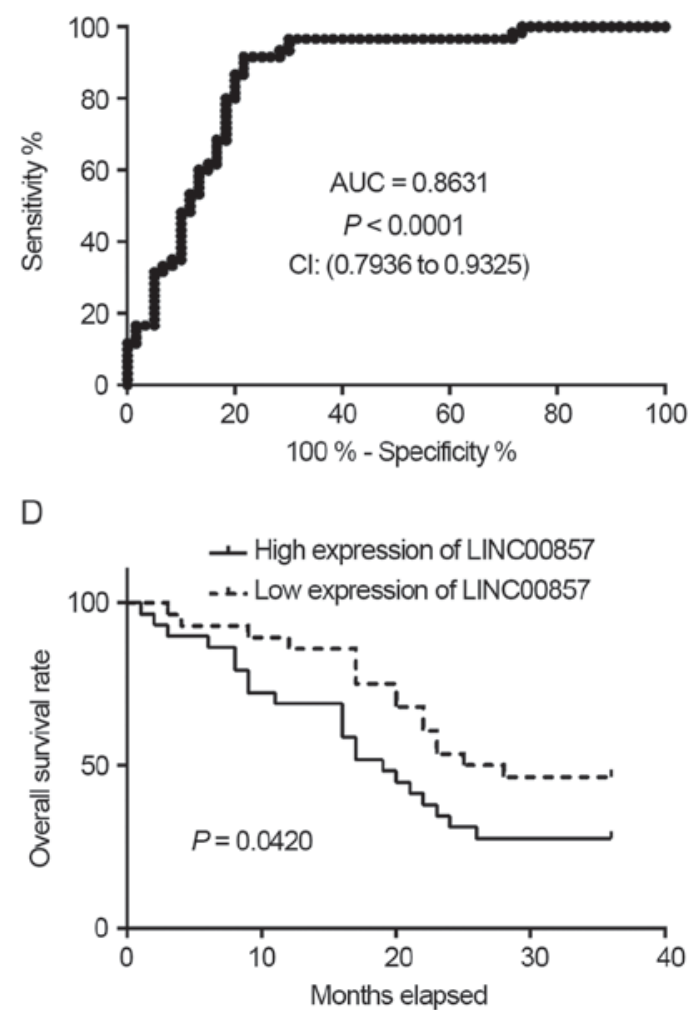

Figure 1. Increased expression of LINC00857 is associated with poor survival rate of patients with GC. (A) LINC00857 expression in 60 pairs of GC tissues and adjacent normal tissues was determined using the reverse transcription-quantitative polymerase chain reaction. (B) ROC curve analysis in GC and non-tumor tissues. (C) Association between LINC00857 expression and tumor size. (D) Kaplan-Meier estimator analysis revealed that patients with GC with increased expression of LINC00857 have a poor survival rate. GC, gastric cancer; ROC, receiver operating characteristics; CI, confidence interval; AUC, area under the curve.

\section{Results}

Increased expression of LINC00857 is associated with poor survival rate in patients with GC. To investigate LINC00857 expression in GC, 60 pairs of GC tissues and adjacent non-tumor tissues were collected. RT-qPCR experiments demonstrated that LINC00857 expression was significantly increased in GC tissues compared with non-tumor tissues $(\mathrm{P}<0.0001$; Fig. 1A). Additionally, receiver operating characteristic (ROC) curve analysis indicated that LINC00857 expression signature exhibited an increased area under the curve (AUC) value of 0.8631 with a sensitivity of $91.67 \%$ and a specificity of $78.33 \%$ in GC tissues (Fig. 1B). Further analysis revealed that LINC00857 expression was positively associated with tumor size (Fig. 1C). Furthermore, survival rate analysis indicated that patients with GC with increased expression of LINC00857 exhibited a poorer survival rate compared with those with low expression of LINC00857 (Fig. 1D). These results suggest that LINC00857 expression may serve as a novel biomarker for the prognosis of GC.

Downregulation of LINC00857 suppresses GC cell proliferation. To determine the function of LINC00857 in the development of GC, certain GC cell lines, including GES-1, AGS, BGC-823, MKN-45 and SGC-7901, were used. RT-qPCR experiments revealed that LINC00857 expression was significantly increased in MKN-45 and SGC-7901 cells compared with that in the remaining cell lines. In particular, the lowest expression of LINC00857 was observed in GES-1 cells (Fig. 2A). Since the highest expression of LINC00857 was observed in MKN-45 and SGC-7901 cells, those cell lines were selected for the subsequent gene transfection experiments as described previously (14). RT-qPCR experiments indicated that the two sets of siRNAs significantly knocked down the expression of LINC00857 in MKN-45 and SGC-7901 cells (Fig. 2B). Cell proliferation was analyzed using a CCK-8 assay and the results indicated that LINC00857 downregulation significantly inhibited the proliferative capacity of MKN-45 and SGC-7901 cells (Fig. 2C and D), suggesting that LINC00857 may promote GC development.

LINC00857 activates multiple signaling pathways involved in the cell cycle. To investigate the molecular mechanisms by which LINC00857 knockdown suppresses cell proliferation, a whole transcriptome analysis using Affymetrix ST2.1 exon arrays was performed in LINC00857-knockdown SGC-7901 cells. SGC-7901 cells transfected with a negative control were used additionally. A hierarchical clustering analysis demonstrated the aberrant expression of multiple transcripts between the two groups (Fig. 3A). Pathway analysis revealed that LINC00857 led to a significant inactivation of the cell cycle signaling pathway (Fig. 3B). RT-qPCR and western blot analysis confirmed that the mRNA and protein expression 
A

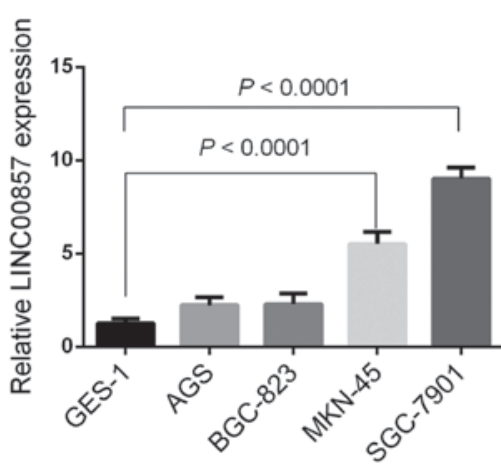

C

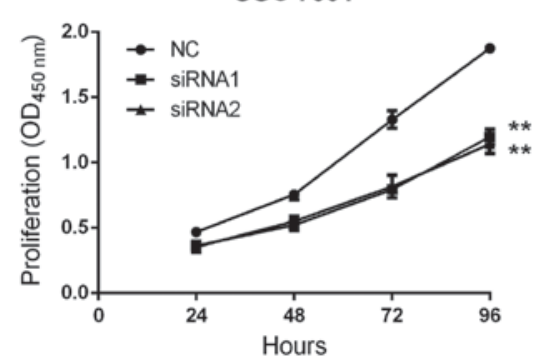

B

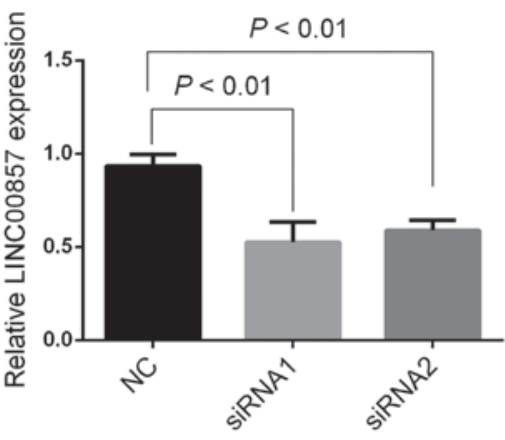

D

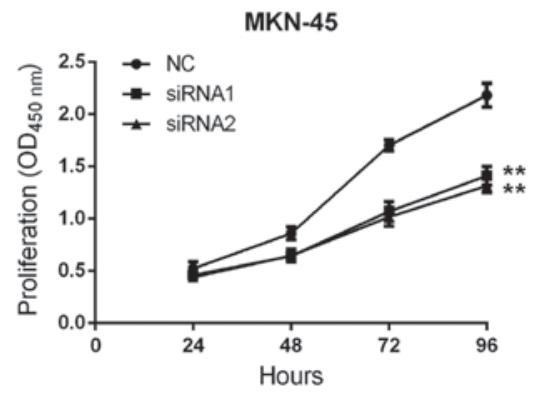

Figure 2. Downregulation of LINC00857 expression inhibits GC cell proliferation. (A) LINC00857 expression in GES-1, AGS, BGC-823, MKN-45 and SGC-7901 GC cell lines was examined using RT-qPCR. (B) LINC00857 expression in SGC-7901 cells was detected using RT-qPCR. (C) Cell proliferation in SGC-7901 cells following transfection with two sets of siRNAs (siRNA1 and siRNA2) was analyzed using the CCK-8 assay. (D) Cell proliferation in MKN-45 cells following transfection with two sets of siRNAs (siRNA1 and siRNA2) was analyzed using the CCK-8 assay. ${ }^{* *} \mathrm{P}<0.01$. GC, gastric cancer; RT-qPCR, reverse transcription-quantitative transcriptase polymerase chain reaction; siRNA, small interfering RNA; CCK-8, Cell Counting Kit-8.

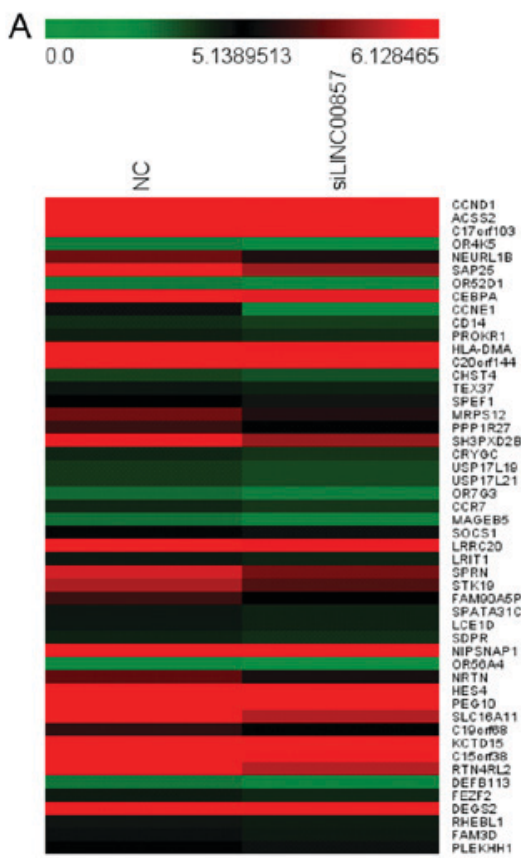

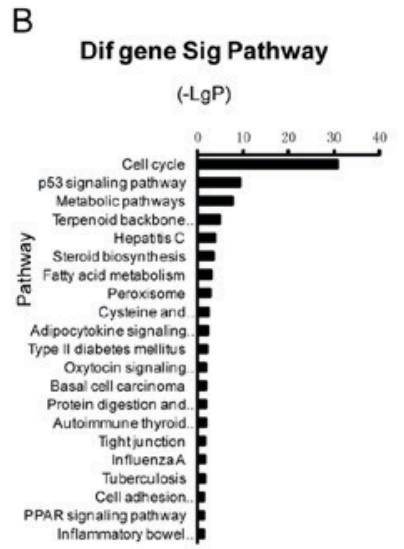

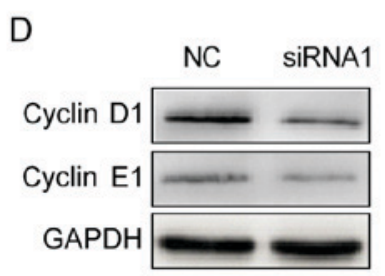

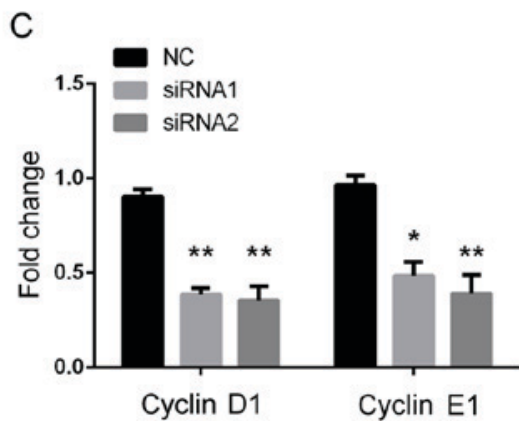

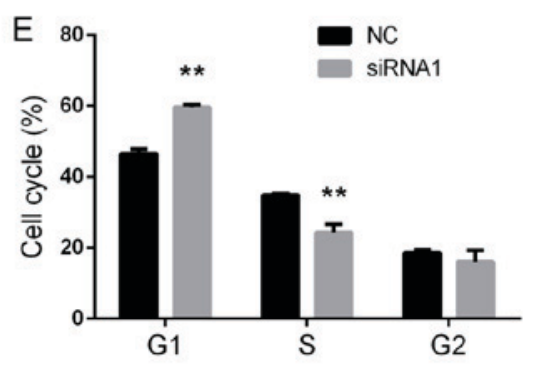

Figure 3. Downregulation of LINC00857 expression arrests GC cells in $\mathrm{G}_{1}$ phase. (A) Genome-wide mRNA expression in SGC-7901 cells following transfection with a control or siRNA1 was analyzed by using microarray. (B) Pathway analysis indicates that downregulation of LINC00857 expression deregulates the cell cycle in SGC-7901 cells. (C) Reverse transcription-quantitative polymerase chain reaction analysis of mRNA expression of cyclin D1 and cyclin E1 in SGC-7901 cells following transfection with two sets of siRNAs (siRNA1 and siRNA2). (D) Western blot analysis of cyclin D1 and cyclin E1 protein expression in SGC-7901 cells following transfection with a control or siRNA1. (E) Flow cytometry of cell cycle analysis in SGC-7901 after transfection with a control or siRNA1. ${ }^{* *}<0.01 ;{ }^{*} \mathrm{P}<0.05$. GC, gastric cancer; siRNA, small interfering RNA.

of cyclin D1 and cyclin E1 were significantly decreased in LINC00857-knockdown SGC-7901 cells compared with in control cells (Fig. 3C and D). Furthermore, flow cytometric analysis indicated that LINC00857-knockdown SGC-7901 
cells were arrested in $\mathrm{G}_{1}$ phase (Fig. 3E). These data suggest that LINC00857 is able to regulate $\mathrm{G}_{1} / \mathrm{S}$ transition in $\mathrm{GC}$ cells.

\section{Discussion}

In the present study, it was demonstrated that increased expression of LINC00857 was associated with the poor survival rate and tumor size of patients with GC. ROC analysis revealed that LINC00857 expression may serve as an independent biomarker for the diagnosis of GC. A cell proliferation assay revealed that knockdown of LINC00857 significantly inhibited the proliferative capacity of GC cells in vitro. Furthermore, genome-wide analysis demonstrated that LINC00857 knockdown resulted in the inactivation of the cell cycle signaling pathway. RT-qPCR and western blot analysis also confirmed that the mRNA and protein expression of cyclin D1 and cyclin E1 were decreased in LINC00857-knockdown SGC-7901 cells. Furthermore, flow cytometric analysis indicated that knockdown of LINC00857 inhibited $\mathrm{G}_{1} / \mathrm{S}$ transition in SGC-7901 cells.

Recent evidence suggests that a number of lncRNAs were able to serve as potential biomarkers for GC (16-19). In a previous study by Zhang et al (13), IncRNA microarray profiling was applied to identify several novel lncRNAs, including LINC00857, for the diagnosis of GC. These data are consistent with the results of the present study in that LINC00857 exhibited an increased AUC value in GC tissues compared with that of non-tumor tissues. The association between LINC00857 expression and the survival rate of patients with GC was further assessed, and it was demonstrated that patients with GC with increased expression of LINC00857 exhibited a poor survival rate. These results suggest that LINC00857 expression may serve as a biomarker for the prognosis of GC.

Although a number of lncRNAs have been annotated, functional interpretation remains limited. Currently, the biological effect of LINC00857 on GC development remains largely unclear. The results of the present study demonstrated that LINC00857 knockdown significantly inhibited GC cell proliferation through regulating cell cycle signaling pathways, confirming the results of previous studies (14). Furthermore, LINC00857 expression might be regulated by cyclin D1 and cyclin E1, which induces $\mathrm{G}_{1}$ arrest in $\mathrm{GC}$ cells. Previous studies have also demonstrated that the downregulation of cyclin D1 and cyclin E1 significantly inhibited GC cell proliferation $(20,21)$. Nevertheless, the molecular mechanisms by which LINC00857 regulates GC cell proliferation require investigation in future studies. Future in vivo experiments are also required to confirm the results of the present study.

In conclusion, the results of the present study demonstrated that LINC00857 expression is associated with the poor survival rate of patients with GC and that downregulating LINC00857 expression suppresses GC cell proliferation in vitro through cell cycle arrest.

\section{Acknowledgements}

The authors thank Professor Zhao Yun for providing support for the reverse transcription-quantitative polymerase chain reaction technology.

\section{Funding}

The present study was supported by the Yongchuan Science \& Technology Commission (grant nos. 2014rc9004 and 2013nc8017).

\section{Availability of data and materials}

The datasets used and/or analyzed during the current study are available from the corresponding author on reasonable request.

\section{Authors' contributions}

FH designed the present study and wrote the manuscript. FH and KP performed the experiments and analyzed the data. MJR, FWZ and TWY collected the samples of the patients and analyzed the data.

\section{Ethics approval and consent to participate}

Ethical approval was obtained from the Ethics Review Board at the Yongchuan Hospital of Chongqing Medical University (Chongqing, China).

\section{Consent for publication}

The authors declare that the patients have provided written informed consent for the publication.

\section{Competing interests}

The authors declare that they have no competing interests.

\section{References}

1. Siegel RL, Miller KD and Jemal A: Cancer statistics, 2016. CA Cancer J Clin 66: 7-30, 2016.

2. Chen W, Zheng R, Baade PD, Zhang S, Zeng H, Bray F, Jemal A, Yu XQ and He J: Cancer statistics in China, 2015. CA Cancer J Clin 66: 115-132, 2016.

3. Karimi P, Islami F, Anandasabapathy S, Freedman ND and Kamangar F: Gastric cancer: descriptive epidemiology, risk factors, screening, and prevention. Cancer Epidemiol Biomarkers Prev 23: 700-713, 2014.

4. Hartgrink HH, Jansen EP, van Grieken NC and van de Velde CJ: Gastric cancer. Lancet 374: 477-490, 2009.

5. Yang AP, Liu J, Lei HY, Zhang QW, Zhao L and Yang GH: CA72-4 combined with CEA, CA125 and CA19-9 improves the sensitivity for the early diagnosis of gastric cancer. Clin Chim Acta 437: 183-186, 2014.

6. Zeng S, Xiao YF, Tang B, Hu CJ, Xie R, Yang SM and Li BS: Long noncoding RNA in digestive tract cancers: Function, mechanism, and potential biomarker. Oncologist 20: 898-906, 2015.

7. Gupta RA, Shah N, Wang KC, Kim J, Horlings HM, Wong DJ, Tsai MC, Hung T, Argani P, Rinn JL, et al: Long non-coding RNA HOTAIR reprograms chromatin state to promote cancer metastasis. Nature 464: 1071-1076, 2010.

8. Tseng YY, Moriarity BS, Gong W, Akiyama R, Tiwari A Kawakami H, Ronning P, Reuland B, Guenther K, Beadnell TC, et al: PVT1 dependence in cancer with MYC copy-number increase. Nature 512: 82-86, 2014.

9. Sun TT, He J, Liang Q, Ren LL, Yan TT, Yu TC, Tang JY, Bao YJ, Hu Y, Lin Y, et al: LncRNA GClncl promotes gastric carcinogenesis and may act as a modular scaffold of WDR5 and KAT2A complexes to specify the histone modification pattern. Cancer Discov 6: 784-801, 2016. 
10. Sun M, Nie F, Wang Y, Zhang Z, Hou J, He D, Xie M, Xu L, De W, Wang $Z$ and Wang J: LncRNA HOXA11-AS promotes proliferation and invasion of gastric cancer by scaffolding the chromatin modification factors PRC2, LSD1 and DNMT1. Cancer Res 76: 6299-6310, 2016.

11. Prensner JR, Iyer MK, Balbin OA, Dhanasekaran SM, Cao Q, Brenner JC, Laxman B, Asangani IA, Grasso CS, Kominsky HD, et al: Transcriptome sequencing across a prostate cancer cohort identifies PCAT-1, an unannotated lincRNA implicated in disease progression. Nat Biotechnol 29: 742-749, 2011

12. Iyer MK, Niknafs YS, Malik R, Singhal U, Sahu A, Hosono Y, Barrette TR, Prensner JR, Evans JR, Zhao S, et al: The landscape of long noncoding RNAs in the human transcriptome. Nat Genet 47: 199-208, 2015.

13. Zhang K, Shi H, Xi H, Wu X, Cui J, Gao Y, Liang W, Hu C, Liu Y, Li J, et al: Genome-wide lncRNA microarray profiling identifies novel circulating lncRNAs for detection of gastric cancer. Theranostics 7: 213-227, 2017.

14. Wang L, He Y, Liu W, Bai S, Xiao L, Zhang J, Dhanasekaran SM, Wang Z, Kalyana-Sundaram S, Balbin OA, et al: Non-coding RNA LINC00857 is predictive of poor patient survival and promotes tumor progression via cell cycle regulation in lung cancer. Oncotarget 7: 11487-11499, 2016.

15. Livak KJ and Schmittgen TD: Analysis of relative gene expression data using real-time quantitative PCR and the $2^{-\Delta \Delta C \mathrm{~T}}$ method. Methods 25: 402-408, 2001.
16. Zhou M, Guo M, He D, Wang X, Cui Y, Yang H, Hao D and Sun J: A potential signature of eight long non-coding RNAs predicts survival in patients with non-small cell lung cancer. J Transl Med 13: 231, 2015.

17. Hu Y, Wang J, Qian J, Kong X, Tang J, Wang Y, Chen H, Hong J, Zou W, Chen Y, et al: Long noncoding RNA GAPLINC regulates CD44-dependent cell invasiveness and associates with poor prognosis of gastric cancer. Cancer Res 74: 6890-6902, 2014.

18. Zhu X, Tian X, Yu C, Shen C, Yan T, Hong J, Wang Z, Fang JY and Chen H: A long non-coding RNA signature to improve prognosis prediction of gastric cancer. Mol Cancer 15: 60, 2016

19. Xia H, Chen Q, Chen Y, Ge X, Leng W, Tang Q, Ren M, Chen L, Yuan D, Zhang Y, et al: The IncRNA MALAT1 is a novel biomarker for gastric cancer metastasis. Oncotarget 7: 56209-56218, 2016.

20. Li P, Chen H, Chen S, Mo X, Li T, Xiao B, Yu R and Guo J: Circular RNA 0000096 affects cell growth and migration in gastric cancer. Br J Cancer 116: 626-633, 2017.

21. Leung SY, Ho C, Tu IP, Li R, So S, Chu KM, Yuen ST and Chen X: Comprehensive analysis of $19 \mathrm{q} 12$ amplicon in human gastric cancers. Mod Pathol 19: 854-863, 2006.

This work is licensed under a Creative Commons Attribution-NonCommercial-NoDerivatives 4.0 International (CC BY-NC-ND 4.0) License. 